http://jmscr.igmpublication.org/home/ ISSN (e)-2347-176x ISSN (p) 2455-0450 crossref DOI: https://dx.doi.org/10.18535/jmscr/v8i10.14

\title{
Lipomatous Pleomorphic Adenoma: A Rare Case Report
}

Authors

\section{Dr Vaishali A Nandkhedkar ${ }^{1}$, Dr Jyoti D Bhavthankar ${ }^{2}$, Dr Mandakini S Mandale, Dr Jayanti G Humbe ${ }^{4}$, Dr Savita P Wagh ${ }^{5}$}

${ }^{1}$ Assistant Professor, Department of Oral Pathology and Microbiology, Government Dental College \& Hospital Aurangabad Maharashtra

${ }^{2}$ Professor and Head, Department of Oral Pathology and Microbiology, Government Dental College \& Hospital Aurangabad Maharashtra

${ }^{3}$ Associate Professor, Department of Oral Pathology and Microbiology, Government Dental College \& Hospital Aurangabad Maharashtra

${ }^{4}$ Associate Professor, Department of Oral Pathology and Microbiology, Government Dental College \& Hospital Aurangabad Maharashtra

${ }^{5}$ Assistant Professor, Department of Oral Pathology and Microbiology, Government Dental College \& Hospital Aurangabad Maharashtra

\section{Abstract}

Pleomorphic adenoma (P A) is a benign salivary gland tumour characterized by cellular and architectural pleomorphism presented as histological heterigenity with chondroid, myxoid or osseous components. Lipomatous differentiation is a rarely observed in PA. Here we are presenting a case report of PA with striking lipomatous differentitation, located in upper lip. Recognition of such rare subtype of PA is important for clinical management. As it is located in upper lip should be considered for the differential diagnosis of Adnexal tumour.

Keywords: PA, LPA, Adnexal tumour, Salivary gland tumour.

\section{Introduction}

Salivary gland tumour constitute an influential area in the field of the pathology .It is important to recognize that neoplasms may arise not only from major salivary glands but also from any of the numerous, diffuse, minor salivary glands. Pleomorphic adenoma is most common salivary gland neoplasm and represents $45-75 \%$ of all salivary gland tumours. Annual incidence is approximately 2-3.5 cases per 10,000 population. About $80 \%$ of pleomorphic adenomas arise in the parotid gland, $10 \%$ in the submandibular gland, and $10 \%$ in the minor salivary gland. Among minor salivary glands accounts, hard palate is the most common site approximately 50-60\%, followed by upper lip (15-20\%) and buccal mucosa $(8-10 \%)^{1}$

Pleomorphic adenoma (PA) is the most common salivary gland neoplasm and yet it has been deemed to be the most difficult SGT in terms of setting the descriptive criteria. Classically, pleomorphic adenoma is characterized by predominance of ductal ,myoepithelial cells along with foci of chondro-myxoid and hyaline stromal development ${ }^{2}$. It's pleomorphism is chalked up to the proliferation of basal and myoepithelial cells 
in a capricious stroma matrix of mucoid, myxoid, cartilaginous or hyaline origin. Along with these routine stromal components PA has been known to show some unusual tissue differentiation in the form of osseous, lipomatous and adnexal areas. ${ }^{3}$ Rarely , lipomatous change is seen in pleomorphic adenoma of minor salivary gland. A PA is designated as Lipomatous pleomorphic adenoma (LPA) in presence of more than 90\% lipomatous stromal component. ${ }^{4} \mathrm{We}$ report one such case of PA of upper lip which exhibited extensive lipomatous metaplasia which is extremely rare variant of pleomorphic adenoma of minor salivary gland.

\section{Case Report}

A 30 year old male patient reported to the Department of oral pathology and microbiology with complain of painless swelling in the upper lip since last 8 months. Past medical history was not conclusive. Clinical examination revealed, small localized swelling in upper lip near the philtrum more towards the skin and intra orally presents as mucosal swelling, round to ovoid in shape measuring approximately $2 \times 1 \mathrm{~cm}$ in size. (fig $1 \& 2$ ). On palpation, the swelling was softfirm, nodular, well circumscribed, freely movable and non-tender. A provisional clinical diagnosis of minor salivary gland neoplasm was made with a differential diagnosis of benign adnexal tumor.

Routine hematological investigations were within normal limits .surgical excision was done with all aseptic precautions.

On macroscopic examination, a lobulated, ovoid mass measuring about $1.5 \times 1 \mathrm{~cm}$ in diameter was received. The cut surface of the resected lesion was smooth and yellowish in color. (fig.4)

Histopathological finding showed a fibrous connective tissue capsule partially enclosing the lesional tissue and the lesional tissue was composed of basal and myoepithelial cells arranged in sheets, strands and nests. Stromal tissue exhibited abundant adipose tissue. Adipocytes were typically univacuolar and did not show cellular atypia. Focal areas of myxo- chondroid and hyaline deposition were also noted (fig.5\&6). Histopathology was consistent with pleomorphic adenoma. Taking into consideration, the extensive presence of adipose tissue, in pleomorphic adenoma labeled as lipomatous variant of pleomorphic adenoma was made. Excision of the lesion had already been done and the patients was followed up for 18 months without any evidence of recurrence.

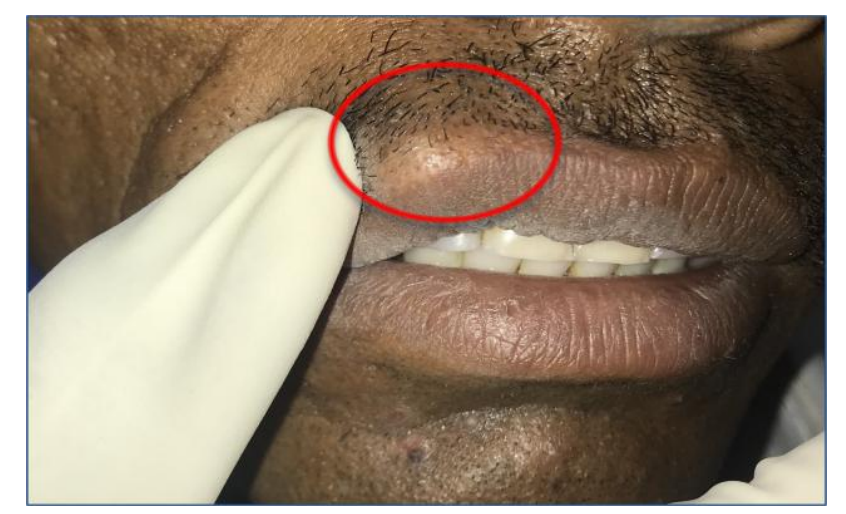

Fig: small round to ovoid swelling seen in the upper lip

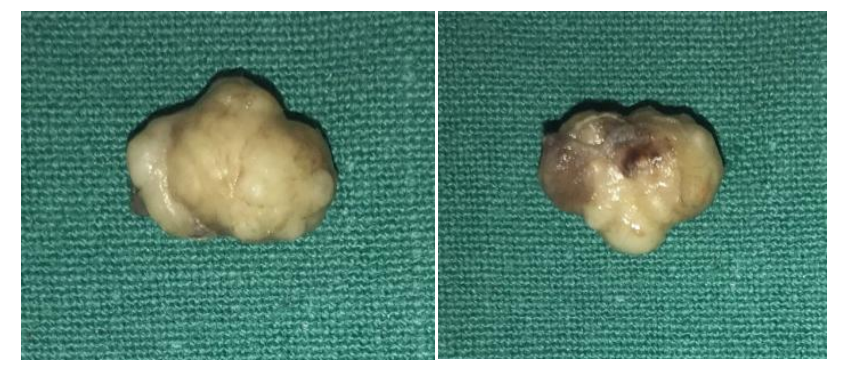

C

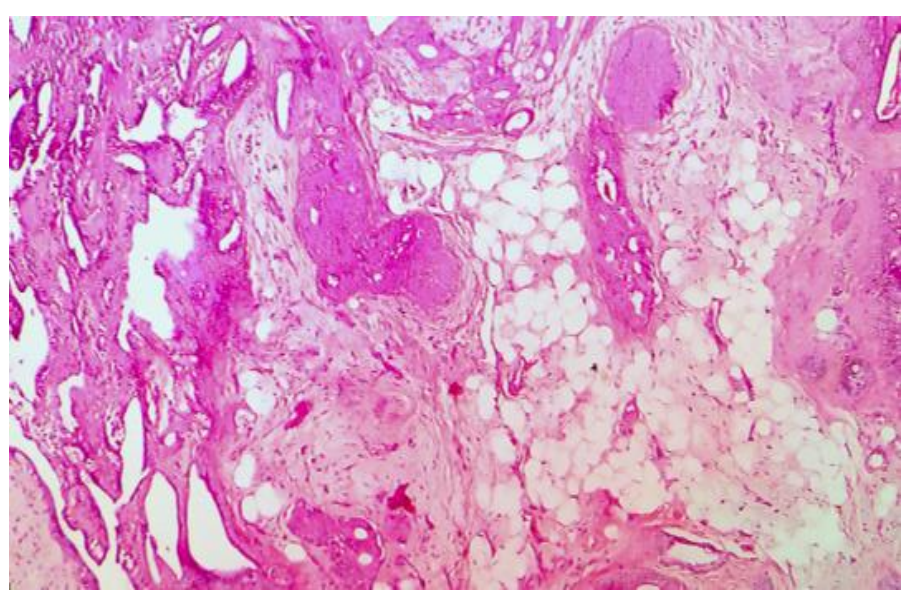

Fig: 10x view 


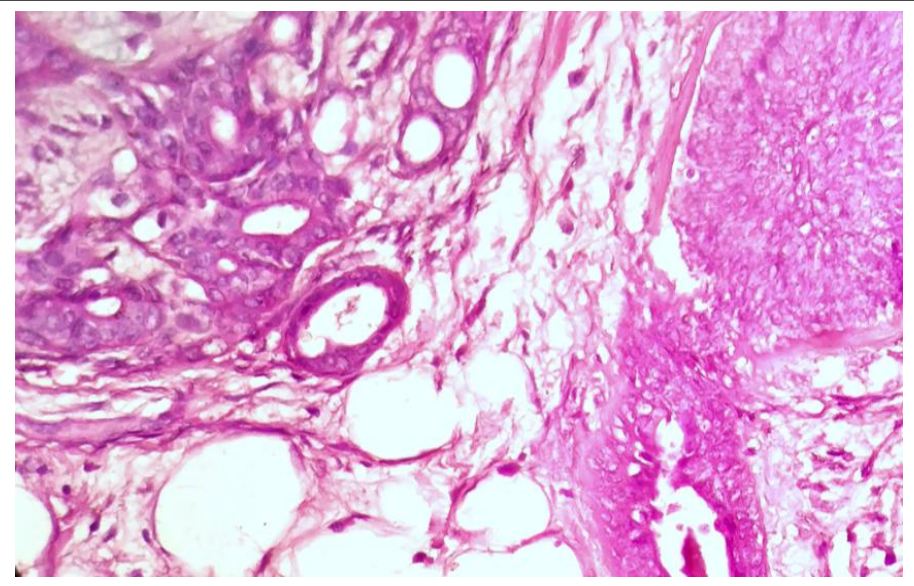

Fig :- 40x view showing the epithelial islands and adipocytes

\section{Discussion}

Pleomorphic adenoma is the most common neoplasm of minor salivary giands, and classically consists of proliferation ductal and myoepithelial cells in chondromyxoid stroma. Other stromal components hyaline or fibrous stroma are found to be rare. But extremely rare variant shows osseous or lipomatous changes in the stroma. Pleomorphic adenomas with an extensive lipomatous stromal component are unusaland very rare histological variant. There are very few reports in the literature, shows pleomorphic adenoma with lipomatous variants. Jamal Musayer et al reported first case of lipomatous pleomorpic adenoma. ${ }^{5}$ The term lipomatous pleomorphic adenomawas coined by Seifert et al. in 1999. Was described by characterizing the tumour as atypical with more than $90 \%$ of its area composed of adipose components. ${ }^{6}$ Various forms of lipomatous tissue overtones within salivary glands have been defined such as lipoma, interstitial lipomatosis, lipoadenoma, oncocytic lipoadenoma, sialolipoma, and lipomatous atrophy. Granting that foci of adipose tissue are occasionally found within the stroma of pleomorphic adenoma, such an extensive replacement by adipose tissue like the current case is quite an unusual finding. ${ }^{6}$

Seifert et al showed that the fat-containing salivary gland tumors can be classified into two main groups based on their histopathological composition: monophasic true adipocytic neoplasms (lipoma and its variants) and hybrid lipoepithelial lesions composed of epithelial variants mixed with a variable fatty component. Alternative acknowledged classification divides fat-containing tumors and tumor-like lesions of salivary glands into 4 categories

1. Fat containing epithelial/myoepithelial tumors such as pleomorphic adenoma;

2. Mixed lipoepithelial tumors such as sialolipoma;

3. True adipose tumors like lipoma;

4. Fat-containing tumor-like salivary gland lesions in which diffuse lipomatosis falls. ${ }^{7}$

Sialolipoma is a benign tumour consisting of adipose tissue admixed with variable amount of adenomatous gland.The tumour present as usually slowly growing mass involving major salivary gland. sialolipoma composed adipose cells and proliferated glandular tissue.

Two possible mechanisms for histogenesis of LPA are suggested-

First doctrine states that the myoepithelial cells shows transformation to adipocytes. Pleomorphic adenoma is also termed as mixed tumor which composes of both epithelial and mesenchymal tissue components. These mesenchymal tissues may be produced by modified Myoepithelial cells which also have great potential to undergo metaplasia. The second revolves around possible entrapment of fat tissue within the growing tumor. ${ }^{4}$

The demographic data pertaining to LPA is scanty owing to its rare occurrence. Jamal et al in their case report have recounted mean age for reported cases, and also including our case to be 37.83 years. The gender distribution is $2: 1$, in favour of female population. Commonly involved site is major salivary gland and in only $10 \%$ cases show involvement of minor salivary gland. Hard palate is the most frequently reported site for LPA of minor salivary gland. Our case presents upper lip involvement which is an unusual location for LPA. For clinical differential diagnosis adnexal tumour is considered. Lipoma was also thought as one of the tumour for differential diagnosis. Another salivary gland tumour that is associated 
with adipose tissue is the lipoadenoma or sialolipoma. It is a well circumscribed tumour that may be encapsulated and consist of adipose tissue and glandular elements including ductal, acinar, basal, and Myoepithelial cells, closely resembling normal salivary gland tissue. Although these tumour have both adipose component and epithelial elements, they differ in lipoadenomas lack the abnormal Myoepithelial cells, chondromyxoid stroma, and mesenchymal metaplasia. $^{2}$.Spindle cell lipoma also considered in the differential diagnosis of a spindle cell lesion showing prominent lipocytic component . spindle cell lipomas may be distinguished from Myoepithelial lesions by their characteristic ropy collagen and an inflammatory infiltrate including large number of mast cells. Another condition such as lipomatousatropy, a condition associated with old age considered as one of the differentiating lesion. In this condition no true mass or capsule is present, but normal salivary structure are intermixed with adipose tissue.In present case, upper lip is the site invoivement, therefore differentiated from the other lesions such as adenexal tumour (chondrosyringoma).

Microscopically, presence of metachromatically stained chondromyxoid stroma and epithelial/myoepithelial component indicative of PA is mandatory. Alternative stains such as Romanowsky type and alcohol-fixed dyes, could be applied to aid in differential diagnosis. Painless swelling in upper lip, should be clinically differentiated from the other lesions commonly occurring in that area such aslipoma, fibroma, mucocele, adnexal tumours such as chondroidsyringoma. Chondroidsyringoma of skin is essentially identical to some of mixed tumour. Chondroidsyringoma are composed of both epithelial and mesenchymal components with sweat gland elements set in a cartiligenous stroma. Literature review suggests that five histological criteria for diagnosis of chondromyxoid syringoma 1) nests of cuboidal cells 2)intercommunicating tubuloalveolar structures lined with two or more rows of cuboidal cells 3) ductal structures composed of one or two rows of cuboidal cells 4) occasional keratinous cysts 5) a matrix of varying composition .chondroidsyringomas may have all five characteristics or manifest only some. Chondroidsyringoma share similarities with pleomorphic adenomas ,but thought to be arise from sweat glands. ${ }^{18}$

\section{Conclusion}

Pathologist should be aware of this rare variant of pleomorphic adenoma. As the site of the lesion in the present case is upper lip near philtrum, may mimick for the adenaxal tumours. Treatment modality is being same for the all variants of pleomorphic adenoma. Literature reveals that high recurrence $8-45 \%$ in pleomorphic adenoma. But the lipomatous variant does not show any recurrence. This rare subtype of pleomorphic adenoma must be known to pathologist in order to establish differential diagnosis, proper treatment modality related with this rare variant of plemorphic adenoma.

\section{References}

1. MNH Khandkar, S M A Sadat, T. Rahman et al. clinical presentation and histological variation of pleomorphic adenoma of salivary giands, Journal of Bangladesh college of Physician and surgeons vol. 37, no.2, April 20190

2. Dardick I. pleomorphic adenoma (Benign mixed tumour ) Text of Salivary Gland Tumour Pathology, Igaku Shoin, Tokyo 1996,75-92

3. Mahmood Jahagir Nezhad, Saedeh Atarbashimaghden, Sepidemokhtari et al Different Histopathological feature of pleomorphic adenoma in Salivary glands Inter. Journal of oral and Maxillofacial Pathology 2013, 4(2), 7-11

4. V.M. Palma, L.M. Barin ,F.M. Pillusky et al pleomorphic adenoma with lipomatous metaplasia in hard palate:rare case report journl of oral and maxillofacial surgery, 
medicine and pathology $\mathrm{xxx} 2017630$ no of 4 .

5. J. Musayev, B. Önal, A. Hasanov, and I. Farzaliyev, "Lipomatous pleomorphic adenoma in the hard palate :report of a rare case with cytohisto correlation and review J. Cytol. 2014 , 31:36-9 (database)

6. G. Seifort, K. Donath and R.Schafer Lipomatouspleomorhic adenoma of the parotid gland . classification of lipomatous Tissue in Salivary Glands Pathol.Res. pract.195:247-252 (1999)

7. Sonal shah and Tamer ZayedMoustafa Case report - An unusal variants of common palatal salivary gland tumour , case report $\mathrm{s}$ of pleomorphic adenoma with significant lipomatous metaplasia . case reports in dentistry vol. 2018, Article Id 2052347, 5 page

8. A. Agaimy, "Fat-containing salivary gland tumors: a review," Head and Neck Pathology, vol. 7, Supplement 1,pp. 90-96 2013

9. H. D. Haskell, K. M. Butt, and S. B. Woo, "Pleomorphic adenomawith extensive lipometaplasia: report of three cases,"The American Journal of Surgical Pathology, vol. 29, no. 10,pp. 1389-1393, 2005.

10. T. Kondo, "A case of lipomatous pleomorphic adenoma in theparotid gland: a case report," Diagnostic Pathology, vol. 4,no. 1, p. 16, 2009.

11. F. Ide and K. Kusama, "Myxolipomatous pleomorphic adenoma:an unusual oral presentation," Journal of Oral Pathology\& Medicine, vol. 33, no. 1, pp. 53-55, 2004

12. F.Ide, A.Tanaka, k. kusama CASE REPORT Further evidence for adipocytic differentiation by the neoplastic myoepithelium. J. Oral patho Med (2007) 36:187-9
13. X. Leroy, A. Wacrenier, D. Augusto, E. Leteurtre, A. Desaulty, and B. Gosselin, "Lipomatous pleomorphic adenoma of theparotid," Annales de Pathologie, vol. 22, no. 3, pp. 219-221,

14. K. Kiciński, B. Mikaszewski, and C. Stankiewicz, "Risk factorsfor recurrence of pleomorphic adenoma" Otolaryngologia Polska,vol. 70, no. 3, pp. 1-7, 2016.

15. Kenichi Matsuzaka, Hideki Fukumoto, etal Lipomatous Pleomorphic adenoma in Palatine Gland Oral Med Pathl(2003)140

16. Ng W K and Ma L. Pleomorphic adenoma with extensive lipoetaplasia. Histopathology 1995 , 27,285-8

17. Siddaraju N Singh N Muniraj F, Jothilingum P.kumar et al. Pre operativecytodiagnosis of pleomorphic adenoma with extensivelipometaplasia a case report ACTA CYTOL 2009, 53,

18. Sonti Sulochana, Madavan Manoha ran, Anitha. Chondroid Syringoma -An unusal presentation. Journal Of Clinical and diagnostic Research 2014, Jul 8 (7) FD13-FD 14. 